\title{
A Chebyshev Polynomial Rate-of-Convergence Theorem for Stieltjes Functions
}

\author{
By John P. Boyd
}

\begin{abstract}
The theorem proved here extends the author's previous work on Chebyshev series [4] by showing that if $f(x)$ is a member of the class of so-called "Stieltjes functions" whose asymptotic power series $\sum a_{n} x^{n}$ about $x=0$ is such that

$$
\varlimsup_{n \rightarrow \infty} \frac{\log \left|a_{n}\right|}{n \log n}=r,
$$

then the coefficients of the series of shifted Chebyshev polynomials on $x \in[0, a], \Sigma b_{n} T_{n}^{*}(x / a)$, satisfy the inequality

$$
\frac{2}{r+2} \geqslant \varlimsup_{n \rightarrow \infty} \frac{\log \left|\left(\log \left|b_{n}\right|\right)\right|}{\log n} \geqslant 1-\frac{r}{2} .
$$

There is an intriguing relationship between this theorem and a similar rate-of-convergence theorem for Padé approximants of Stieltjes functions which is discussed below.
\end{abstract}

A Stieltjes function is defined by the integral

$$
f(x)=\int_{0}^{\infty} \frac{\rho(t) d t}{1+x t}
$$

where the weight function $\rho(t)$ is such that

$$
\rho(t) \geqslant 0, \quad t \in[0, \infty],
$$

and all the moment integrals

$$
a_{n}=\int_{0}^{\infty} t^{n} \rho(t) d t
$$

exist for all nonnegative integers $n$ [3]. Although special, this class of functions plays a major role in nuclear, atomic, and elementary particle theory, in critical phenomenon, and in a variety of other fields as shown in the books by Baker [2], Graves-Morris [8], [9], and Cabannes [6]. One reason for the great attention given to the Stieltjes functions is that one can prove the convergence of the Pade approximants formed from their power series. The purpose of this brief note is to show that one can prove a rate-of-convergence theorem for the Chebyshev polynomial series of these functions which is similar to-and intriguingly related to-that already known for Padé approximants.

By expanding $(1+x t)^{-1}$ in (1), using the binomial theorem, and then integrating term-by-term, one can show that the coefficients of the power series expansion of

Received March 17, 1981; revised November 18, 1981.

1980 Mathematics Subject Classification. Primary 42A56; Secondary 33A65, 41A10.

Key words and phrases. Chebyshev polynomial series. 
$f(x)$ are $(-1)^{n} a_{n}$, where the $a_{n}$ are defined by the moment integrals (3). The power series is only an asymptotic series, however, because $f(x)$ has a branch point singularity at $x=0$.

In a similar way, by using the known Chebyshev expansion [11] of $(1+x t)^{-1}$, one can derive

$$
f(x)=\sum_{n=0}^{\infty} d_{n}(-1)^{n} b_{n} T_{n}^{*}(x),
$$

where $d_{0}=1, d_{n}=2$ for $n \geqslant 1$, where the $T_{n}^{*}(x)$ are the shifted Chebyshev polynomials defined on the interval $[0,1]$, and where

$$
b_{n}=\int_{0}^{\infty} \frac{1}{(1+t)^{1 / 2}} q^{n} \rho(t) d t,
$$

where

$$
q(t)=1-\frac{2}{t}\left[(1+t)^{1 / 2}-1\right] .
$$

The similarities between (5) and (3) are striking: both the power series and Chebyshev coefficients are defined by integrals involving the product of $\rho(t)$ with a function of $t$ raised to the $n$th power. However, whereas the power series is only asymptotic and the binomial expansion of $(1+x t)^{-1}$ is convergent only for $t<1 / x$, one can show that the Chebyshev series of $f(x)$ converges faster than any finite inverse power of $n$ and also that the Chebyshev expansion of $(1+x t)^{-1}$ is convergent for any finite $t$. The proof that the Chebyshev series of $f(x)$ converges exponentially on $[0,1]$, despite the singularity at $x=0$, follows from a simple integration-by-parts argument [7] that depends only on the boundedness of all derivatives of $f(x)$ at the singular point, which for the Stieltjes functions is guaranteed by the existence of the moment integrals (3).

Our goal is to relate the magnitude of the Chebyshev coefficients $b_{n}$ to the magnitude of the asymptotic series coefficients $a_{n}$, working through the integral representations of these coefficients, (3) and (5). To do this, let us write

$$
b_{n}=\int_{0}^{\infty} B(t) d t
$$

split the integral into two via

$$
\begin{aligned}
& I_{1}=\int_{0}^{n^{\alpha}} B(t) d t, \\
& I_{2}=\int_{n^{\alpha}}^{\infty} B(t) d t,
\end{aligned}
$$

where $\alpha$ is a cutoff that will be chosen later, and then prove separate bounds on each integral.

LEMma 1. If the integral $I_{1}$ is defined by (8), (7), and (5), then

$$
I_{1} \leqslant f(0) e^{-0.828 n^{(1-\alpha / 2)}}
$$

for $\alpha>0$. 
Proof. It is easy to show that $q(t)$, as defined by (6), is a monotonically increasing function of $t$. In consequence,

$$
\begin{aligned}
I_{1} & =\int_{0}^{n^{\alpha}} \frac{1}{(1+t)^{1 / 2}} q^{n} \rho(t) d t \\
& \leqslant\left[q\left(n^{\alpha}\right)\right]^{n} \int_{0}^{n^{\alpha}} \rho(t) d t .
\end{aligned}
$$

Since $\rho(t)$ is nonnegative, the integral in (12) is obviously bounded by the corresponding integral with an upper limit of $\infty$, which is $a_{0}=f(0)$ according to (3). We can in turn put a simple bound on $q^{n}\left(n^{\alpha}\right)$ by writing

$$
q^{n}=e^{n \log [1-T]}
$$

where

$$
T=\frac{2}{t}\left[(1+t)^{1 / 2}-1\right] .
$$

Identity 4.1 .34 of [8] shows that

$$
e^{n \log [1-T]}<e^{-n T}
$$

for all positive $n$ and $T$. Since the ratio

$$
\frac{T(t)}{P / t^{1 / 2}}=\frac{2}{P t^{1 / 2}}\left[(1+t)^{1 / 2}-1\right],
$$

where $P$ is a positive constant is monotonically increasing with $t$ for any $t>1$ and is $>1$ for $P<2\left[2^{1 / 2}-1\right]$, it follows that we can replace $T$ on the right-hand side of (15) by $P / t^{1 / 2}$ without disturbing the inequality if $P \leqslant 2\left[2^{1 / 2}-1\right]=0.828$. Replacing $t$ in (14) and (15) by $n^{\alpha}$ and then substituting (15) into (12) then gives the lemma.

LEMMA 2. If the integral $I_{2}$ is defined by (9), (7), and (5), then, for $\alpha>0$,

$$
I_{2} \leqslant e^{-n \alpha \log (n)} a_{n},
$$

where the $a_{n}$ are the power series coefficients defined by (3).

Proof. By multiplying and dividing the integrand by $t^{n}$, the definition of $I_{2}$ gives

$$
I_{2}=\int_{n^{\alpha}}^{\infty} \frac{(q / t)^{n} t^{n} \rho(t) d t}{(1+t)^{1 / 2}} .
$$

Since $q(t)<1$ for all positive $t$ and $(1 / t)$ is monotonically decreasing,

$$
I_{2} \leqslant \frac{1}{\left(1+n^{\alpha}\right)^{1 / 2}\left[n^{\alpha}\right]^{n}} \int_{n^{\alpha}}^{\infty} t^{n} \rho(t) d t .
$$

Since the integrand is identical with that which defines the power series coefficient $a_{n}$ and since $\rho(t)>0$, it follows that

$$
I_{2} \leqslant n^{-\alpha n} a_{n}
$$

which is the lemma.

Together, the two lemmas show that

$$
b_{n} \leqslant e^{-0.828 n^{(1-\alpha / 2)}} f(0)+e^{-n \alpha \log (n)} a_{n}
$$


for any positive $\alpha$. The remaining step is to choose $\alpha$ in such a way that the bound (21) is as tight as possible.

To do this, it is helpful to define a measure of how rapidly the $a_{n}$ are growing with $n$, as done previously in Boyd [4]:

Definition. The order $r$ of the asymptotic series $\sum a_{n} x^{n}$ about $x=0$ is defined by

$$
r \equiv \varlimsup_{n \rightarrow \infty} \frac{\log \left|a_{n}\right|}{n \log (n)} .
$$

Thus, the series $\sum(-1)^{n} n ! x^{n}$ has order 1 , while $\sum(-1)^{n}(2 n) ! x^{n}$ and $\sum(-1)^{n}(n !)^{2} x^{n}$ both have $r=2$. The greater the order of the series, the more rapidly the $a_{n}$ increase with $n$.

In a similar way, again following Boyd [4], we have

Definition. The index of exponential convergence $\beta$ of the series $\sum b_{n} T_{n}^{*}(x)$, where the $T_{n}^{*}(x)$ are the shifted Chebyshev polynomials defined on $x \in[0,1]$, is the least upper bound of those $k$ for which

$$
\left|b_{n}\right| \leqslant p e^{-q n^{k}}
$$

can be satisfied for all $n$ with some finite positive constants $p$ and $q$.

We can now state the following

THEOREM. If $f(x)$ is a Stieltjes function with an asymptotic power series about $x=0$ whose convergence order is $r$, as defined by (22) above, then the index of exponential convergence $\beta$ of its shifted Chebyshev polynomial series on $x \in[0,1]$, as defined by (23), must satisfy

$$
\frac{2}{r+2} \geqslant \beta \geqslant 1-\frac{2}{r+\varepsilon}
$$

where $\varepsilon>0$ may be arbitrarily small.

The theorem can be extended to Chebyshev expansion intervals $[0, a]$ by making the trivial change of variable $x \rightarrow(x / a)$.

Proof. The left inequality in (23) is proved in Boyd [4] and is in fact valid for any function, not necessarily a Stieltjes function, which is singular at $x=0$ but has an asymptotic power series about $x=0$ so that all its derivatives there are bounded.

The right inequality can be proved by setting

$$
\alpha=r+\varepsilon
$$

in (21). The definition of the order, (22), then guarantees that, in the limit $n \rightarrow \infty$, the second term in (21) is exponentially small $(O[\exp \{-n \varepsilon \cdot \log (n)\}])$ in comparison to the first. Neglecting this second term then gives the theorem.

One can state the theorem in somewhat looser terms as follows: if the power series coefficients $a_{n} \sim(n !)^{r}$ for large $n$, then the Chebyshev series coefficients $b_{n}$ must converge at least as fast as the sequence $\exp \left[-n^{(1-2[r+\varepsilon])}\right]$, where $\varepsilon$ is an arbitrarily small positive number. The author's previous work [4] has put an upper bound on the rate of convergence of the Chebyshev polynomial series for functions which have asymptotic power series about one endpoint of the expansion interval. The theorem proved here shows that, for Stieltjes functions, a lower bound on the rate of Chebyshev convergence is also possible. 
There is an intriguing relationship between the Chebyshev theorem derived here and previously known theorems for Padé approximants of Stieltjes functions, despite the fact that these two methods of approximating a function are seemingly very different.

Bender and Orszag [3] note, rephrasing their results in terms of the language used here, that if a Stieltjes function is of order $r$, then, writing the Pade approximants as continued fractions, the maximum difference between the $n$th continued fraction $F_{n}(x)$ and the next highest continued fraction $F_{n+1}(x)$ on a finite interval in $x$ is bounded by

$$
\max _{x \in[0,1]}\left|F_{n}(x)-F_{n+1}(x)\right|<e^{-p n^{[1 / 21}}
$$

for some positive constant $p$. Since the continued fractions correspond to partial sums of a series, the difference between two adjacent continued fractions is directly analogous to the series coefficient $b_{n+1}$. Thus, (26) is equivalent to the statement that, for continued fractions, the index of convergence is $\beta=1-r / 2$ precisely - the same as the lower bound on the Chebyshev index of convergence.

This relationship between Chebyshev series and Padé approximants also exists for functions which have no singularities on the expansion interval. One can show, for example, that the error in the continued fraction at a point $z$ for the function $f(z)=z^{-1} \ln (1+z)$ decreases geometrically with $n$ at a rate identical with that of the Chebyshev series on the interval $[0, z]$ for the same function where $z$ may be complex. (The Padé approximants for this function are discussed in Bender and Orszag [3] and the Chebyshev theory for a function with a simple pole or branch point in Gottlieb and Orszag [7].) Obviously, the relationship between Padé approximants and Chebyshev series deserves further exploration. Luke [10] has already made a beginning.

It is easy to show by a specific example, however, that one cannot replace the right inequality in (24) by an equals sign nor naively assume that Chebyshev and Padé methods will always give identical accuracy. The Stieltjes function, the prototype of the class of the Stieltjes functions, is defined by

$$
f(x)=\int_{0}^{\infty} \frac{e^{-t}}{1+x t} d t .
$$

It is shown in Luke [10] that the Chebyshev coefficients satisfy

$$
b_{n} \sim O\left[e^{-3 n^{2 / 3}}\right],
$$

so that the index of convergence $\beta=2 / 3$ as is given by equality in the left inequality in (24). In contrast, the difference between adjacent continued fractions for this same function satisfy

$$
F_{n}-F_{n+1} \sim O\left[e^{-p n^{1 / 2}}\right]
$$

for some positive constant $p$ for fixed $x$. Thus, for the Stieltjes function, Chebyshev polynomials are vastly superior to Padé approximants.

An obvious goal of future work is therefore to determine if the theorem proved here can be sharpened to perhaps show that $\beta=2 /(r+2)$ exactly at least for some subclass of the Stieltjes functions. One powerful method of deriving asymptotic 
expressions for the Chebyshev coefficients of an individual function is by using the method of steepest descents as in Luke [11] and Boyd [5]. It may well be that, for density functions $\rho(t)$ which are smooth enough so that steepest descents can be applied, $\beta=2 /(r+2)$ always. However, the density function $\rho(t)$ can be very nonsmooth-even a distribution. Since Chebyshev series are very sensitive to the smoothness of the function being expanded, it may well be that Stieltjes functions, whose density functions $\rho(t)$ are distributions, have $\beta=1-r / 2$, so that (24) is the best one can do for the class as a whole.

Acknowledgment. This work was supported by NASA Grant NGL-22-007-228 at Harvard and NSF Grant OCE-79-09191 at Michigan. I am grateful to Professor Richard Lindzen for his hospitality at Harvard, where much of this work was performed.

Department of Atmospheric and Oceanic Science

University of Michigan

Ann Arbor. Michigan 48109

1. J. Abramowitz \& I. Stegun, Eds., Handbook of Mathematical Functions, Dover, New York, 1965.

2. (j. Baker, JR., Essentials for Pade Approximants, Academic Press, New York, 1975.

3. C. Bender \& S. A. Orszag, Advanced Mathematical Methods for Scientists and Engineers, McCiraw-Hill, New York, 1978.

4. J. BOYD. "The rate of convergence of Chebyshev polynomials for functions which have asymptotic power series about one endpoint," Math. Comp., v. 37, 1981, pp. 189-195.

5. J. BoYD. "The optimization of convergence for Chebyshev polynomial methods in an unbounded domain,”J. Comput. Phis., 1982. (To appear.)

6. H. Cabannes, Ed., Pade Approximants Method and Its Application to Mechanics, Springer-Verlag, Berlin, 1973.

7. D. Gottlieb \& S. A. Orszag, Numerical Analysis of Spectral Methods: Theory and Applications, SIAM, Philadelphia, Pa., 1977.

8. P. Graves-Morris, Ed., Padé Approximants, Institute of Physics, London, 1973.

9. P. Graves-Morris, Ed., Padé Approximants and Their Applications, Academic Press, New York, 1973.

10. Y. L. LUKE, “Chebyshev expansions and rational approximations," J. Comput. Appl. Math., v. 2. 1976. pp. 85-95.

11. Y. Luke, The Special Functions and Their Approximations, Vol. I, Academic Press, New York, 1969. 